Vol. 2 No. 3, 2021

\title{
THE METHODOLOGY OF EVALUATION OF THE WORKPLACE DIGITALIZATION LEVEL
}

\author{
Dariia Doroshkevych ${ }^{1}$, Inna Lytvynenko
}

\begin{abstract}
The purpose the worldwide increase of the digitalization level and COVID-19 pandemic at the same time lead to a situation in which companies have to adapt to the trend of remote economics and change the working environment of their employees. Methodology. This research aimed at improving the methodology for assessing the level of workplace digitalization and to evaluate the level of workplace digitalization on the example of a company "IT marketing", taking into account technical and moral factors. Recent years statistics confirms the growth of people working from home and building careers remotely. Increasing the level of mobility and flexibility of personnel, digitization of the workplace, national and gender diversity of staff - all these trends proof that the workforce is a powerful factor for the intensive growth of the whole company. But at the same time the process of workplace's digitizing requires deep scientific researches. Strengthening the level of security, the presence of digital etiquette, new corporate culture, the moral stress of staff to keep in touch can lead to burnout and reduce employee's performance and therefore - profitability of enterprises. It is impossible to ignore the trend towards increasing remote economics that is proved by numerous scientific studies. At the same time, sociologists argue that today for the effective functioning of the economy the vector must be shifted from technology to humans. One of the main objectives of the presented research is to identify tools that can increase the level of digitalization of the workplace and at the same time enlarge the efficiency of working staff. These tools should cover all aspects of doing business including customer and employee engagement, accounting and financial transactions, project management and productivity. Thus, the presented article reflects the study of current trends in the socio-economic sphere, the results of which may have an impact on human relations in companies and meet the needs of employees while observance of high level of their work efficiency. Results. The research we used our methodology and evaluated the level of digitalization on the example of company "IT-Marketing". Practical implications. In the article gave the recommendations to the company's management how to increase the level of workplace digitalization.
\end{abstract}

Key words: digital workplace, workplace digitalization, digitalization, digital economy, remote economy.

JEL Classifications: $\mathrm{O33}$

\section{Introduction}

Jobs have changed rapidly in recent years. For new work environments, organisations, employers and employees need to adapt to digitalization in order to be constantly productive and not lose customers.

The concept of the digital workplace has grown in popularity, as an old understanding of the fact that a workplace is somewhere, in a specific place where employees spend physical time, is no longer relevant.

The world, which has already become digital, requires new tools for establishing cooperation with its workers. This raises the question of the need for a combination of communications, ideas and digital systems, which will stimulate working order and become an effective hub for the exchange of information among all

\footnotetext{
Corresponding author:

${ }^{1}$ International University of Finance, Ukraine

National Technique University of Ukraine "KPI", Ukraine

University of Economy in Bydgoszcz, Poland

Poznan University of Natural Sciences, Poland

E-mail: dariya.dor@gmail.com

ORCID: https://orcid.org/0000-0003-0421-0630

${ }^{2}$ International University of Finance, Ukraine

E-mail: ilytvynenko1995@gmail.com

ORCID: https://orcid.org/0000-0002-3954-0202
} 
participants. This is what will be the digital workplace.

The digital world is full of content, information and tools that make collaboration and management a challenge. Combining communications, ideas and systems to maintain order and serve as a hub for effective information exchange is what makes your digital workplace.

The main benefits of digitising the workplace are improving productivity, cost-effectiveness, and workers who are quickly adaptable and skilful. Employees will need to develop new skills and meet new challenges.

Employers need to invest in the training of their employees to ensure that the workforce is qualified for digitised jobs. At the same time, it is important not only to understand the importance of the introduction of digital jobs, but also to realistically assess the level of digitalization of the workplace, as well as the willingness of people to employ new forms of communication and work without tight control. That is why the topic of this article, especially in the conditions of the remote economy development, is relevant and corresponds to the realities of today.

We have decided to combine the views of different scholars into one methodology that includes both technical factors and factors of moral preparedness.

The first group of factors of the presented methodology are the factors that will help to evaluate the level of automation of business processes. The next stage of our methodology is moral readiness for remote work. The third stage, as well as the first, covers the technical aspects. It concerns the security and reliability of the digital workplace. The last fourth stage is a logical continuation of the third one.

\section{Research materials and methods}

The emerging digital workplace, considered the natural evolution of the workplace, can address the issues listed above and help organisations (Kruse and Geißler, 2015; Menkens, 2016).

Frank Schönefeld (2011) defines the digital workplace as the "totality of the required access infrastructure, applications and device platforms of information or knowledge workers who need them to perform their work tasks and engage in collaboration".

The success of introducing digital workplaces depends on how well a digital location strategy is implemented. As each workplace strategy, a digital one is supposed to fulfil business objectives such as reducing property costs, improving business performance, merging two or more organisations/cultures, and relocating or consolidating occupied buildings. The workplace strategy and its implementation quite often occur at an opportune moment such as a property lease break or a company merger or acquisition (Hoffmeister and Yorck, 2015; Kampschroer et al, 2017).

According to (EU, 2016; Hoffmeister and Yorck, 2015; Savage, 2005; Springer, 2011), the reasons for devising and adopting the digital workplace strategy are talent attraction, new communication tools, employee productivity, satisfaction and more stable retention.

Thought by Deloitte (2011), a proposal for a digital workplace framework is presented with the layers such as use, technology, control and business drivers. According to Hamburg (2018), the components of the digital workplace governance are guiding principles, information governance, roles and responsibilities, training and certification, policy training, orchestrated presence and crisis management. All of these stages are sequential and interdependent. Thus, neglecting only one of the steps presented may cause the digital workplace to function ineffectively.

One can agree with the opinion of CMSWire (2014) regarding the digital workplace that "the digital workplace is meant to be a virtual equivalent to the physical workplace". There is a similar opinion of Clearbox (2015) according to which, the digital workplace "is the concept that there is a virtual equivalent to the physical workplace, and that this needs to be planned and managed coherently because it is fundamental to people's productivity, engagement and working health."

The choice of methods for investigation of workplace digitalization level is conditioned by the ambiguity of its notion in the economic literature. We have concluded to accept definitions of CMSWire (2014), Clearbox (2015) and Deloitte (2011), according to which digital workplace must have benefits such as attracting talent, employee productivity, employee satisfaction, retention of employees, communication and collaboration tools.

Remote management is a method of managing business, as well as other areas of human life, in 
which management becomes possible without the direct involvement of the "leader", but with the possibility of feedback. Referring to the previously published publications on this topic, we can say that companies with remote management are becoming more widespread around the world - from virtual small business assistants to teams of large companies such as IBM, to digital start-ups with fully remote teams. According to Global Workplace Analytics, remote teams increase employee productivity, satis-faction levels, and can save a company more than $\$ 10,000$ annually. That is not including the benefit of tapping into a global talent pool (Nevogt, 2019).

John Stevenot (2017) wrote about a debate among scientists about the distinction between a remote and flexible job. Remote work is considered to be performed solely outside the office. The place does not matter, it is only important to fulfil one's work obligations. In this case, flexible work corresponds to the situation where an employee can independently choose their place of work and the same flexible schedule, which does not depend on the geographical location of the employee. Nonetheless, flexible work, like remote work, involves performing certain tasks within a certain amount of time.

The issue of digital workplace security deserves special attention. According to Copyright, 2018, the security features governing the security of an identity can be divided into three levels of security. These three levels of security, in combination, provide comprehensive security for protecting personal information in important documents and ensuring the originality and accuracy of certificates.

However, only some scientists, in particular (Hanna and Mona, 2014; Maslach, 2003) point to the need to take into account institutional settings, occupational stress that can stem from a complex combination of actual work-related conditions or factors, interpersonal stressors, such as disagreements with co-workers or supervisors, or environmental conditions, including inadequate support or pressure to complete tasks, international etiquette, protocol consultancy, business and corporate etiquette, cross cultural awareness, expats social life skills in the Middle East, image \& attire, finishing school, personal development, digital and social media. According to (Tarver, 2019), corporate culture refers to the beliefs and behaviour that determine how a company's employees and management interact and handle outside business transactions.

Thus, it can be concluded that in order to assess the level of job digitalization, different types of factors should be taken into account, including safety indicators, digital equipment, employees' morale and others.

The Harvard Business Review magazine conducted a staff survey in (2018) and concluded that workers around the world are seeking freedom and flexibility. The most common form of flexibility offered by companies is the ability to work remotely. A new study (Pulse, 2018) found that one-third of workers worldwide always or frequently work remotely. Compared to a decade ago, the number of remote workers increased by $115 \%$. The study also declared that only $5 \%$ always or very often see themselves in their company throughout their careers, compared to almost a third who never work remotely.

Based on the theory and methodology of evaluating the level of workplace digitalization, we ask several research questions: (1) What are the main factors that must be included in the methodology? (2) How to evaluate these factors? (3) Can our methodology help to increase the level of workplace digitalization in the terms of remote economy?

For evaluation the workplace digitalization we used survey method created questionnaire with variants of answers, where each answer correspond with defined grades. Grades correlate with the significance of every answer. All employees involved in the process of remote work answered the questions. Based on this information we created the coefficient that indicates the level of each factor. Correlation analysis helped us to understand the cross connections between all stages and factors. As a result, we proposed the methodology based on analytical methods with the use of indicators for digital workplace evaluation and correlation analysis to make the conclusions. This methodology can be transferred to all companies that use remote workers with any quantity of employees in it.

\section{Research results}

An analysis of the literature has shown that the focus of the researchers is mainly on the technical aspects of assessing the level of workplace digitalization. Moreover, they all highlight the 


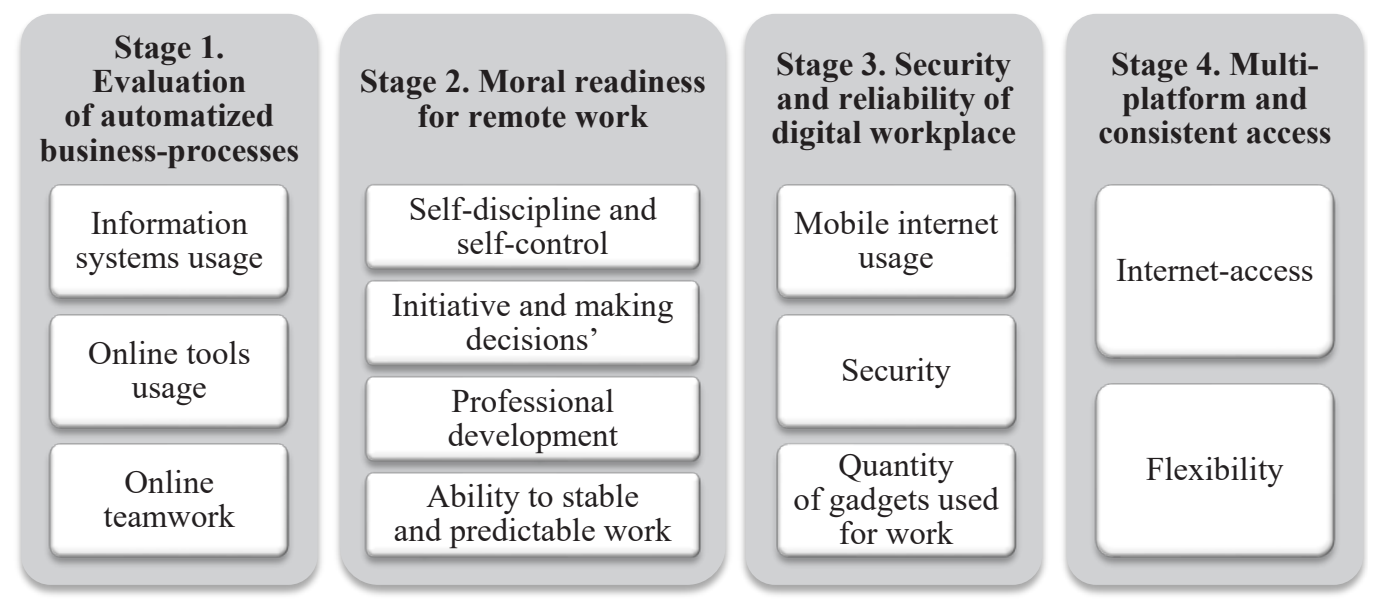

Figure 1. Methodology of the level of workplace digitalization

factors that have a greater impact on the digital workplace. We have decided to combine the views of different scholars into one methodology that includes both technical factors and factors of moral prepared-ness (Figure 1).

The first group of factors of the presented methodology are the factors that will help to evaluate the level of automation of business processes. The level of workplace digitalization depends primarily on how actively the staff use information systems to perform their daily tasks. These factors are the result of the use of digital, mainly cloud, technologies, tools and platforms that allow them to organise their digital workplace, providing a convenient way to carry out tasks from anywhere in the world. That is why we have chosen three factors to evaluate the level of business processes automation:

- information system usage;

- online tools usage;

- online team work.

The information system usage factor includes the need to evaluate information cloud system usage and online project management usage. Search engine usage, the ability to use data analytics and digital files and podcasting usage belong to the factor of online tools usage. One of the most important tasks of remote management of employees is to ensure that they perform all their work duties on time, efficiently and in accordance with the existing standards. The issue of business processes automation correlates with the execution of projects and tasks in accordance with the schedule. However, at the same time, a certain number of remote workers work for a certain number of hours per day.
In any case, control over their activities is impossible without the use of information management systems of different types. These tools cover all aspects of doing business, including customer relationship management, human relationship management, accounting, financial and resource management, project and supply chain management. At the same time, cloudtechnologies allow people to collect statistics, including for the efficiency of work of those who have chosen a remote form of work.

More attention should be given to an understanding of teamwork planning. Working with a remote team has the potential to increase productivity only if certain difficulties are overcome. One of such problems may be the remote work of employees living in multiple time zones. When all employees are in the same geographic region, it is easier to set clear rules and boundaries. However, if remote employees work around the world, coordination of their work requires other technical tools and more detailed planning of the work process, which in turn leads to the generation of new competencies for team leaders. One of the vital tasks of managing a remote employee is to ensure that they perform all their work duties on time, efficiently and in accordance with standards.

The third factor in Stage 1 is online teamwork. This factor includes the evaluation of the ability of online discussion used and social media support from colleagues. The need to evaluate these indicators is related to the fact that communication between team members is a crucial factor in organising effective work. Managing a remote team is a complex process, and communication 
can be a big stumbling block to many companies trying to focus on the remote work of employees. That is why the management has to adopt an effective communication strategy so that the feeling of isolation from the team does not affect the progress of employees. Interaction among employees and daily virtual communication create a sense of trust and connection in the team.

The next stage of our methodology is moral readiness for remote work. We have added 4 factors to this stage:

- self-discipline and self-control;

- initiative and making decisions;

- professional development;

- ability to do stable and predictable work.

According to the experts (The Harvard Business Review, 2018), people who have chosen to work remotely face two major moral problems. They are burnout and loneliness. Burnout is a common cause of poor performance. It arises from a lack of willingness to work remotely, lack of self-control and low self-motivation. An unlimited working day, work at the weekends and on vacation days also affect this phenomenon. In addition, management decisions are often made without the employee's opinion. Therefore, in order to prevent the onset of burnout, we suggest periodically evaluating this stage, which will influence the quality of work performed and the degree of use of all technical tools offered to the worker.

At this stage, it should be understood that there are factors that affect the employee's work performance in the digital workplace. These include:

- ineffective work of employees in the absence of a direct manager;

- people's unwillingness to work without direct instructions;

- lack of professional development due to poor communication;

- lack of planning and, as a consequence, unstable and unsystematic work of remote staff.

Based on this, we have included questions that correlate with each factor of Stage 2 in our case study. Particular attention should be paid to assessing staff creativity and innovation. In our opinion, no less important aspect of creating a digital workplace and of its effective functioning is psychology of management. It is the ability of the leader of remote staff to create a favourable psychological climate, the ability to persuade, motivate, share his or her inspiration, lead his or her employees, find and develop their strengths (including - creative ones) and promote further digitalization of the workplace. All these things are possible only if the employee's moral readiness is at a high level.

The third stage, as well as the first, covers the technical aspects. It concerns the security and reliability of the digital workplace.

Remote management hardware is personal computers, smartphones, data protection systems and other workflow support systems. The technical equipment of an organisation, consisting of the above elements, is an integral part, which in general makes it possible to have a digital workplace, providing communication between the executives of a company and its affiliates, managers and staff of these affiliates. In addition, remote work requires additional security measures, and enterprises must consider additional guarantees for protecting the data managed by remote workers using VPN, when connecting to the Internet and using password managers. Organisations will then need to provide new tools and security protocols for remote workforce. As new technologies evolve and the demand for specialised distributed workforce rises, remote work will continue to evolve with them. The new generation of workers determines how and where work is completed. In order to attract and retain the best talent, organisations must continue to learn new ways that their employees use to interact with business and with each other, regardless of zip code.

That is why the factors that characterise this stage comprise:

- mobile internet usage;

- security;

- quantity of gadgets used for work.

To assess the level of security and reliability, we suggest evaluating the level of mobile Internet usage, the ability to find viruses and install security systems without anyone's help, and the number of files downloaded from the Internet. The number of gadgets used for work is also a very important indicator, as a large number of gadgets means increased levels of danger and increased protection of information.

Guaranteeing security is one of the priority tasks facing all organisations that use remote staff. To ensure the implementation of this function, special units and security services are created that 
provide protection for the inside information and interests of a company.

The last fourth stage is a logical continuation of the third one. We propose evaluating the multiplatformity and the availability of constant access to the Internet, without which it is impossible to work remotely and work in the digital workplace. Multiplatformity and quality access are the foundation of an effective digital environment in the digital workplace. We analyse the experience of regular network connection as to how good remote access and stability of the $\mathrm{Wi}-\mathrm{Fi}$ Internet access are, especially in regions. Setting up and solving problems with the infrastructure and access is very important for improving one's digital workplace.

\section{Conclusion}

The paper proposes a methodology for assessing the level of digitalization of the workplace in the conditions of development of a remote economy. The analysis of the literature showed that more attention is paid to technical and safety indicators, neglecting employees' morale and their willingness to work remotely. That is why, we have developed our assessment methodology, which takes into account existing strategies of workplace digitalization. We have divided the methodology into 4 stages and identified key factors at each stage by which we will evaluate the level of job digitalization. One of the stages we offer, namely the stage of moral willingness to work remotely, solves the problem of assessing people's readiness to work according to plan, to feel that they are part of a team, as well as problems of self-discipline and taking the initiative. In order to calculate the level of digitalization, we have conducted a sociological survey based on a real enterprise in the IT industry, where all employees work remotely or have experience of working with remote workers.

According to the conclusion in future scientific researches we must check the relevance of the methodology on an example of a company that use digital workplaces and remote management. It will shows if the given methodology can be used on real enterprises.

We have conducted a sociological survey of the employees of the Ukrainian company "IT-marketing". Each employee has his or her own digital workplace and experience of remote work in various positions: as a contractor or as a man-ager managing remote work. This study will be published in the next part of the article.

The goal is a digital environment that enhances the performance of staff and the mobility of work areas. This will ensure a stable workflow that is sup-ported by consistent access to the $\mathrm{Wi}$-Fi network. If the study reveals a low level of this indicator, such things should be done: improve the equipment, install high quality software and ensure multiplatformity.

The research aimed at improving the methodology for assessing the level of digitalisation of the digital workplace and finding the best options for enhancing indicators is relevant and in line with the realities of today. The digital workplace is the future for most companies in the market and a tool that will help them succeed.

\section{References:}

Deloitte | Audit, Consulting, Financial, Risk Management, Tax Services (2011). The digital workplace: think, share, do transform your employee experience. Available at: https://www2.deloitte.com/ content/dam/Deloitte/mx/Documents/human-capital/The_digital_workplace.pdf

EU (2016). Skills Challenges in Europe. Available at: http://skillspanorama.cedefop.europa.eu/sites/ default/files/2016_Skills_Challenges_AH.pdf

Hamburg, I. (2018). Implementation of a Digital Workplace Strategy to Drive Behavior Change and Improve Competencies. Available at: https://www.intechopen.com/books/strategy-and-behaviors-inthe-digital-economy/implementation-of-a-digital-workplace-strategy-to-drive-behavior-change-andimprove-competencies

Hanna, T., \& Mona, E. (2014). Psychosocial work environment, stress factors and individual characteristics among nursing staff in psychiatric in-patient care. International Journal of Environmental Research and Public Health, 11(1):1161-1175.

Harvard Business Review - Ideas and Advice for Leaders (2018). Remote Workers Are More Disengaged and More Likely to Quit. Available at: https://hbr.org/2018/11/survey-remote-workersare-more-disengaged-and-more-likely-toquit?autocomplete=true

Hoffmeister, C., \& Yorck von B. (2015). Think New! 22 Erfolgsstrategien im digitalen Business. München: Carl Hanser Verlag. ISBN 978-3-446-44228-3 
Kampschroer, K., Heerwagen, J., \& Powell, K. (2017). Creating and testing workplace strategy. California Management Review, 49(2):19-137.

Kruse, P., \& Geißler, P. (2015). Das vernetzte Unternehmen: Wie der Digital Workplace unsere Zusammenarbeit neu gestaltet. Norderstedt: BoD-Books on Demand. ISBN 978-3-7392-9620-3

Maslach, C. (2003). Job burnout: New directions in research and intervention. Current Directions in Psychological Science, 12(5):189-192.

Menkens, S. (2016). Beim Homeoffice ist Deutschland noch Entwicklungsland - WELT. Available at: https://www.welt.de/print/die_welt/politik/article155662124/Beim-Homeoffice-ist-Deutschlandnoch-Entwicklungsland.html

Nevogt, D. (2019). 5 Common Challenges of Managing Remote Employees. Available at: https://blog.hubstaff.com/remote-management-problems

Savage, A. E. (2005). Workplace strategy: What it is and why you should care. Journal of Corporate Real Estate, 7(3).

Schönefeld, F. (2011). Social intranet - The new role of the intranet for the digital workplace. In: Wolf F, editor. Social Intranet - Promoting Communication - Sharing Knowledge - Working Together Efficiently. München: Carl Hanser Verlag, pp. 14-40. ISBN 3-446-42791-0

Springer, T. (2011). Fundamentals of Workplace Strategy. Illinois: Kimball.

Stevenot, J. (2017). What is the difference between telecommuting, work-at-will, remote working and flexible work? Available at: https://medium.com/@johnstevenot/what-is-the-difference-betweentelecommuting-work-at-will-remote-working-and-flexible-work-3e1b3d9d5445

Tarver, E. (2019). Corporate Culture Definition. Available at: https://www.investopedia.com/terms/ $c /$ corporate-culture.asp 